\title{
HALL EFFECT ON MHD TRANSIENT FLOW PAST AN IMPULSIVELY STARTED INFINITE HORIZONTAL POROUS PLATE IN A ROTATING SYSTEM
}

\author{
B. PRABHAKAR REDDY \\ Department of Mathematics, CNMS, University of Dodoma \\ P. Box. No. 338, Dodoma, TANZANIA \\ Department of Mathematics, Geethanjali College of Engineering \& Technology \\ Cheeryal (V), Keesara (M), Medchal (Dist) - 501301, Telangana, INDIA \\ E-mail: prabhakar.bijjula@gmail.com
}

\begin{abstract}
In this paper, the effect of Hall current on an unsteady MHD transient three dimensional flow of an electrically conducting viscous incompressible fluid past an impulsively started infinite horizontal porous plate relative to a rotating system has been studied. It is assumed that the entire system rotates with a constant angular velocity about the normal to the plate and a uniform magnetic field is applied along the normal to the plate and directed into the fluid region. The magnetic Reynolds number is assumed to be so small that the induced magnetic field can be neglected. The expressions for the primary and secondary fields and shearing stress at the plate due to primary and secondary velocity fields are obtained in a non-dimensional form. The non-dimensional governing equations of the flow are solved by using the Galerkin FEM. The effects of the physical parameters, such as the Hartmann number $(\mathrm{M})$, rotation parameter $(\Omega)$, porosity parameter $(K)$ and Hall parameter $(\mathrm{m})$ on primary and secondary velocities and shearing stresses $\tau_{x}$ and $\tau_{y}$ due to primary and secondary velocities are discussed through graphs and tables, and results are physically interpreted.
\end{abstract}

Key words: Hall current, MHD, transient, rotation, horizontal plate, Galerkin FEM.

\section{Introduction}

MHD deals with the motion of an electrically conducting fluid in the presence of a magnetic field. There are numerous examples of application of the MHD principle. The dynamo and motor is a classical example of MHD principle. Geophysics encounters MHD characteristics in the interaction of a conducting fluid and the magnetic field. Engineers apply the MHD principle in fusion reactors, dispersion of metals, metallurgy, design of MHD pumps, MHD generators and MHD flow meters, etc. The MHD principle also finds its application in medicine and biology. Applications in biomedical engineering include cardiac MRI, ECG, etc. The mechanism of conduction in ionized gases in the presence of a strong magnetic field is different from that in a metallic substance. The electric current in ionized gases is generally carried by electrons which undergo successive collisions with other charged or neutral particles. In the ionized gases, the current is not proportional to the applied potential except when the electric field is very weak. However, in the presence of a strong electric field, the electrical conductivity is affected by the magnetic field. Consequently, the conductivity parallel to the electric field is reduced. Hence, the current is reduced to the direction normal to both electric and magnetic fields. This phenomenon is known as the Hall effect. The effect of Hall current on MHD flows has been studied by many researchers due to the application of such studies in the problems of MHD generators and Hall accelerators. Datta and Jana [1] studied the Hall current effects on oscillatory magneto-hydrodynamic flow past a flat plate. Biswal and Sahoo [2] presented the Hall current effects on oscillatory hydro-magnetic free convective flow of a visco-elastic fluid past an infinite 
vertical porous flat plate with mass transfer. Watanabe and Pop [3] presented Hall effects on a magnetohydrodynamic boundary layer flow over a continuous moving flat plate. Aboeldahab and Elbarbary [4] studied the Hall current effect on a magneto- hydrodynamic free convection flow past a semi-infinite vertical plate with mass transfer. The Hall current effect with simultaneous thermal and mass diffusion on an unsteady hydro-magnetic flow near an accelerated vertical plate was studied by Acharya et al. [5]. Sharma et al. [6] presented the Hall effect on MHD mixed convective flow of a viscous incompressible fluid past a vertical porous plate, immersed in a porous medium with heat source/sink. Prabhakar Reddy and Anand Rao [7] presented radiation and thermal diffusion effects on an unsteady MHD free convection mass transfer flow past an infinite vertical porous plate with Hall current and heat source. Raju et al. [8] presented the Hall current effects on an unsteady MHD flow between a stretching sheet and an oscillating porous upper parallel plate with constant suction. Recently, Rajput and Kanaujia [9] studied MHD flow past a vertical plate with variable temperature and mass diffusion in the presence of Hall current.

The rotating flow of an electrically conducting fluid in the presence of a magnetic field is encountered in geographical fluid dynamics. It is also important in the solar physics dealing with the sunspot development, the solar cycle and the structure of rotating magnetic stars. It is well known that a number of astronomical bodies possess fluid interiors and magnetic fields. Changes that take place in the rate of rotation per minute, suggest the possible importance of hydro-magnetic spin-up. Sattar and Maleque [10] studied an unsteady MHD natural convection flow along an accelerated porous plate with Hall current and mass transfer in a rotating porous medium. The MHD flow over a moving plate in a rotating fluid with magnetic field, Hall currents and free stream velocity was studied by Takhar et al. [11]. Mbeledogu and Ogulu [12] studied heat and mass transfer of an unsteady MHD natural convection flow of a rotating fluid past a vertical porous flat plate in the presence of radiative heat transfer. Combined effects of Hall current and rotation on free a convection MHD flow in a porous channel were studied by Singh and Rakesh Kumar [13]. Ahmed and Sarma [14] studied the MHD transient flow past an impulsively started infinite horizontal porous plate in a rotating system with Hall current. The effects of Hall current and radiation absorption on the MHD micropolar fluid in a rotating system were presented by Satyanarayana et al. [15].

The present paper is to analyze the effect of Hall current on an unsteady MHD transient three dimensional flow of an electrically conducting viscous incompressible fluid past an impulsively started infinite horizontal porous plate relative to a rotating system. The Galerkin FEM has been adopted to solve the governing equations of the flow. The behaviors of the primary and secondary velocities, shearing stresses have been discussed and presented for variations in the governing parameters.

\section{Mathematical model}

The equations governing the motion of an incompressible viscous electrically conducting fluid in a rotating system in the presence of a magnetic field are

Equation of continuity

$$
\nabla \cdot q=0
$$

Momentum equation

$$
\rho\left[\frac{\partial \boldsymbol{q}}{\partial t^{\prime}}+2 \boldsymbol{\Omega} \times \boldsymbol{q}+\boldsymbol{\Omega} \times(\boldsymbol{\Omega} \times \boldsymbol{r})+(\boldsymbol{q} \cdot \nabla) \boldsymbol{q}\right]=-\nabla p+\boldsymbol{J} \times \boldsymbol{B}+\mu \nabla^{2} \boldsymbol{q} .
$$

Kirchhoff's first law

$$
\nabla . J=0 .
$$


General Ohm's law

$$
\boldsymbol{J}+\frac{\omega_{e} \tau_{e}}{B_{0}}(\boldsymbol{J} \times \boldsymbol{B})=\sigma\left[\boldsymbol{E}+\boldsymbol{q} \times \boldsymbol{B}+\frac{1}{e \eta_{e}} \nabla p_{e}\right] .
$$

Gauss's law of magnetism

$$
\nabla \cdot \boldsymbol{B}=0
$$

where $\boldsymbol{q}$ is the velocity vector, $\Omega$ is the angular velocity of the fluid, $\boldsymbol{r}$ is the position vector of the fluid particle $P$ considered, $p$ is the pressure, $\boldsymbol{J}$ is the current density, $\boldsymbol{B}$ is the magnetic induction vector, $\boldsymbol{E}$ is the electric field, $2 \Omega \times \boldsymbol{q}$ is the Coriolis acceleration, $\Omega \times(\Omega \times \boldsymbol{r})$ is the centripetal acceleration and the other symbols have their usual meaning.

We consider an unsteady MHD flow of an incompressible viscous electrically conducting fluid past a suddenly started infinite horizontal porous plate relative to a rotating system with constant suction in the presence of a magnetic field taking into account the effect of Hall current. The present investigation is restricted to the following assumptions.

(i) All the properties are constant and the buoyancy force has no effect on the flow.

(ii) The plate is electrically non-conducting.

(iii) The entire system is rotating with angular velocity $\Omega$ about the normal to the plate and $|\Omega|$ is so small that $\boldsymbol{\Omega} \times(\boldsymbol{\Omega} \times \boldsymbol{r})$ can be neglected.

(iv) The magnetic Reynolds number is so small that the induced magnetic field can be neglected.

(v) $p_{e}$ is constant.

(vi) $\boldsymbol{E}=0$

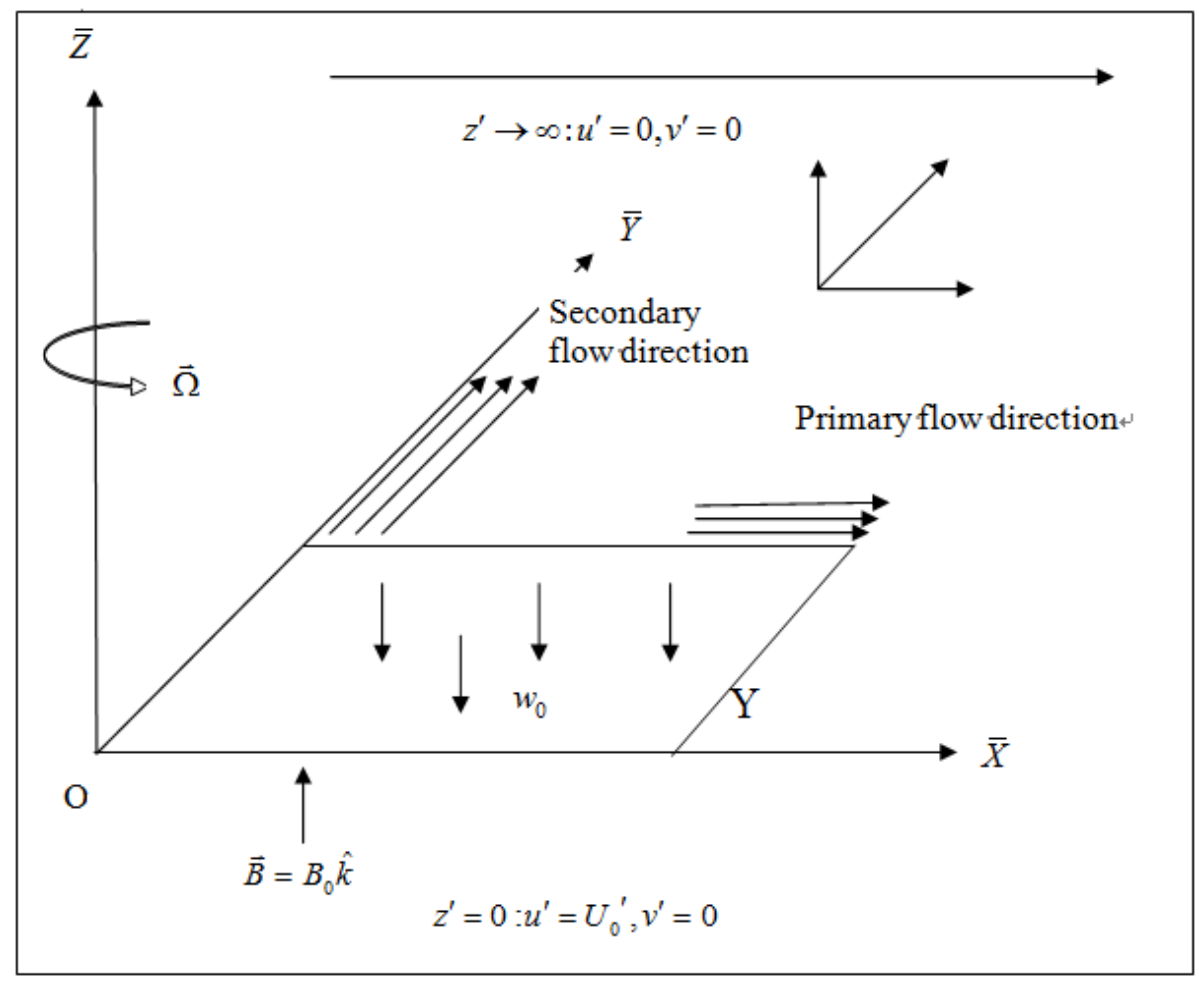

Fig.1. Geometry of the flow. 
Initially, the plate and the fluid were rotating in unison with constant angular velocity $\boldsymbol{\Omega}$ about the normal to the plate. At time $t^{\prime}>0$, the plate is suddenly moved in its own plane relative to the rotating system with velocity $U_{0}^{\prime}$ which is thereafter maintained constant. We introduce the coordinate system $\left(x^{\prime}, y^{\prime}, z^{\prime}\right)$ with the $X$ axis horizontally in the direction of the plate velocity, $Y$ axis horizontally perpendicular to the direction of the plate velocity and $Z$ axis along the normal to the plate which is its axis of rotation. Let $\boldsymbol{q}=u^{\prime} \hat{i}+v^{\prime} \hat{j}+w^{\prime} \hat{k}$ be the fluid velocity, $\boldsymbol{J}=J_{x}^{\prime} \hat{i}+J_{y}^{\prime} \hat{j}+J_{z}^{\prime} \hat{k}$ be the current density at the point $P\left(x^{\prime}, y^{\prime}, z^{\prime}, t^{\prime}\right)$ and $\boldsymbol{B}=B_{0} \hat{k}$ be the applied magnetic field, $\hat{i}, \hat{j}, \hat{k}$ being the unit vectors along the $X$ axis, $Y$ axis and $Z$ axis respectively. As the plate is infinite in the $X$ direction and $Z$ direction, therefore, all the quantities except possibly the pressure are independent of $x^{\prime}$ and $y^{\prime}$.

Equation (2.1) gives $\frac{\partial w^{\prime}}{\partial z^{\prime}}=0$,

which is trivially satisfied by $\bar{w}=-w_{0}^{\prime}=$ a constant

Therefore the velocity vector $\boldsymbol{q}$ is given by

$$
\boldsymbol{q}=u^{\prime} \hat{i}+v^{\prime} \hat{j}-w_{0}^{\prime} \hat{k}
$$

Equation (2.5) is satisfied by $\boldsymbol{B}=B_{0} \hat{k}$.

Equation(2.3) reduces to $\frac{\partial J_{z}^{\prime}}{\partial z^{\prime}}=0$ which gives $J_{z}^{\prime}=0$,

(as the plate is electrically non-conducting)

Hence, the current density is given by

$$
\boldsymbol{J}=J_{x}^{\prime} \hat{i}+J_{y}^{\prime} \hat{k}
$$

Under the assumptions (v) and (vi), Eq.(2.4) takes the form

$$
\boldsymbol{J}+\frac{m}{B_{0}}(\boldsymbol{J} \times \boldsymbol{B})=\sigma(\boldsymbol{q} \times \boldsymbol{B})
$$

where $m\left(=\omega_{e} \tau_{e}\right)$ is the Hall parameter.

Equations (2.8), (2.9), (2.11) and (2.12) yield

$$
\begin{aligned}
& J_{x^{\prime}}=\frac{\sigma B_{0}}{1+m^{2}}\left(m u^{\prime}-v^{\prime}\right), \\
& J_{y^{\prime}}=\frac{\sigma B_{0}}{1+m^{2}}\left(u^{\prime}+m v^{\prime}\right) .
\end{aligned}
$$

With these assumptions and under the usual boundary layer approximation, Eq.(2.2) reduces to 


$$
\begin{aligned}
& \frac{\partial u^{\prime}}{\partial t^{\prime}}-w_{0}^{\prime} \frac{\partial u^{\prime}}{\partial z^{\prime}}-2 \Omega^{\prime} v^{\prime}=v \frac{\partial^{2} u^{\prime}}{\partial z^{\prime 2}}-\frac{\sigma B_{0}^{2}\left(u^{\prime}+m v^{\prime}\right)}{\rho\left(1+m^{2}\right)}-\frac{v u^{\prime}}{K^{\prime}} \\
& \frac{\partial v^{\prime}}{\partial t^{\prime}}-w_{0}^{\prime} \frac{\partial v^{\prime}}{\partial z^{\prime}}+2 \Omega^{\prime} u^{\prime}=v \frac{\partial^{2} v^{\prime}}{\partial z^{\prime 2}}-\frac{\sigma B_{0}^{2}\left(v^{\prime}-m u^{\prime}\right)}{\rho\left(1+m^{2}\right)}-\frac{v v^{\prime}}{K^{\prime}}
\end{aligned}
$$

with $\quad \frac{\partial p^{\prime}}{\partial z^{\prime}}=0$

where $w_{0}^{\prime}$ is the constant suction velocity, $v$ is the kinematic viscosity and the relevant initial and boundary conditions are

$$
\begin{array}{lll}
t^{\prime} \leq 0 ; & u^{\prime}=0, \quad v^{\prime}=0, \quad \text { for all } \quad z^{\prime} \\
t^{\prime}>0 ; & u^{\prime}=U_{0}^{\prime}, \quad v^{\prime}=0 \quad \text { at } \quad z^{\prime}=0, \\
u^{\prime}=0, & v^{\prime}=0 \quad \text { as } \quad z^{\prime} \rightarrow 0 .
\end{array}
$$

We introduce the following non-dimensional variables and parameters

$$
u=\frac{u^{\prime}}{U_{0}^{\prime}}, \quad z=\frac{w_{0}^{\prime} z^{\prime}}{v}, \quad t=\frac{w_{0}^{\prime 2} t^{\prime}}{v}, \quad v=\frac{v^{\prime}}{U_{0}^{\prime}}, \quad M=\frac{\sigma B_{0}^{2} v}{\rho w_{0}^{\prime 2}}, \quad K=\frac{K^{\prime} U_{0}^{2}}{v^{2}}, \quad \Omega=\frac{2 \Omega^{\prime} v}{w_{0}^{\prime 2}}
$$

Using the above non-dimensional quantities in Eqs (2.15), (2.16) and (2.17), we obtain the following non-dimensional form of the governing equations of the flow

$$
\begin{aligned}
& \frac{\partial u}{\partial t}-\frac{\partial u}{\partial z}-\Omega v=\frac{\partial^{2} u}{\partial z^{2}}-\frac{M}{1+m^{2}}(u+m v)-\frac{1}{K} u \\
& \frac{\partial v}{\partial t}-\frac{\partial v}{\partial z}+\Omega u=\frac{\partial^{2} v}{\partial z^{2}}-\frac{M}{1+m^{2}}(v-m u)-\frac{1}{K} v
\end{aligned}
$$

The corresponding initial and boundary conditions are

$$
\begin{array}{llll}
t \leq 0 ; & u=0, & v=0 & \text { for all } \quad z, \\
t>0 ; & u=1, & v=0 \quad \text { at } \quad z=0, \\
u=0, & v=0 \quad \text { as } \quad z \rightarrow 0 .
\end{array}
$$




\section{Method of solution}

Finite element methods are widely used to solve boundary value problems. Here, we use the Galerkin finite element method to solve the governing Eqs (2.18) and (2.19), under the boundary conditions given in Eq.(2.20). The method entails the following steps.

1. Division of the whole domain into smaller elements of finite dimensions called "finite elements".

2. Generation of the element equations using variational formulations.

3. Assembly of element equations as obtained in step 2.

4. Imposition of boundary conditions to the equations obtained in step 3 .

5. Solution of the assembled algebraic equations.

The assembled equations can be solved by any of the numerical technique viz. Gauss-Seidal iteration method. An important consideration is that of shape functions which are employed to approximate actual functions. For one dimensional and two dimensional problems, the shape factions can be linear/quadratic and higher order. However, the suitability of the shape functions varies from problem to problem. Due to simple and efficient use in computations linear shape functions are used in the present problem. The numerical solutions for the primary and secondary velocities are obtained by using $C$ program. To judge the convergence and stability of the Galerkin FEM, the same program was run with slightly changed values of step sizes in the time and space directions, no significant change was observed in the values of the primary velocity $(u)$ and the secondary velocity $(v)$. Hence, we conclude that the Galerkin finite element method is convergent and stable.

\section{Shearing stresses}

The shearing stress at the plate in the direction of the primary velocity is given by

$$
\tau_{x}=-\left(\frac{\partial u}{\partial z}\right)_{z=0}
$$

The shearing stress at the plate in the direction of the secondary velocity is given by

$$
\tau_{y}=-\left(\frac{\partial v}{\partial z}\right)_{z=0}
$$

\section{Results and discussion}

In order to study the effects of physical parameters, such as the magnetic parameter $(M)$, rotation parameter $(\Omega)$, porosity parameter $(K)$ and Hall parameter $(\mathrm{m})$ on the flow, numerical calculations have been carried out for the non-dimensional primary velocity $u$ and secondary velocity $v$ as well as shearing stresses $\tau_{x}$ and $\tau_{y}$ due to primary and secondary velocities, respectively. The obtained numerical results are presented in figures and tables. These results show the effect of the material parameters on the quantities mentioned.

The effects of the magnetic parameter $M$ on the primary and secondary velocity fields are presented in Figs 1 and 2, respectively. It can be seen that an increase in the magnetic parameter decreases the primary velocity and the reverse effect is observed on the secondary velocity. That is retarded under the effect of the transverse magnetic field. This phenomenon is clearly supported by the physical reality. Figures 3 and 4 depict the effect of the Hall parameter $m\left(=\omega_{e} \tau_{e}\right)$ on the primary and secondary velocity fields, respectively. It is seen that an increase in the Hall parameter increases the primary and secondary velocity fields. Also, the effect of the Hall parameter $m$ has a minor increasing effect on the primary velocity whereas 
there is a quite larger increasing effect on the secondary velocity which indicates and also supports the fact that the Hall parameter induces a cross-flow in the boundary layer. The effects of the porosity parameter $K$ on the primary and secondary velocity fields are presented in Figs 5 and 6, respectively. It is observed that an increasing values of the porosity parameter increases in both primary and secondary velocities. Figures 7 and 8 depict the effect of the rotation parameter $\Omega$ on the primary and secondary velocity fields, respectively. It is clear that an increase in the rotation parameter decreases the primary velocity and increases the secondary velocity. Also, we notice that the rotation parameter has a little effect on the primary velocity whereas there is a quite larger increasing effect on the secondary velocity. The same figures further demonstrate that the primary velocity asymptotically decreases from its maximum value $u=1$ to its minimum value $u=0$ as $z$ increases and the secondary velocity first increases in a very thin layer adjacent to the plate and after this layer it asymptotically decreases to zero as $z$ increases. In other words, it may be stated that the secondary motion is accelerated under the effects of the Hall current and rotation due to the application of the transverse magnetic field.

The numerical values of the shearing stresses $\tau_{x}$ and $\tau_{y}$ due to the primary and secondary velocities for variations in the magnetic parameter $(M)$, Hall parameter $(\mathrm{m})$, porosity parameter $(K)$ and rotation parameter $(\Omega)$ are presented in Tab.1. It is seen that an increase in the strength of the magnetic field and angular velocity of rotation of the fluid leads to an increase in $\tau_{x}$ and $\tau_{y}$ falls due to the Hall effect. Also, an increase in the porosity parameter decreases $\tau_{x}$. From this observation, we may interpret that the viscous drag on the plate due to primary motion is reduced under the Hall effect, but this frictional force increases the effect of the magnetic field, rotation of the fluid. The magnetic strength, rotation of the fluid and porosity parameter $\tau_{y}$ increasing. Also, for small and moderate values of the Hall parameter $\tau_{y}$ rises as the Hall parameter increases but for large values of the Hall parameter this behavior takes a reverse trend.

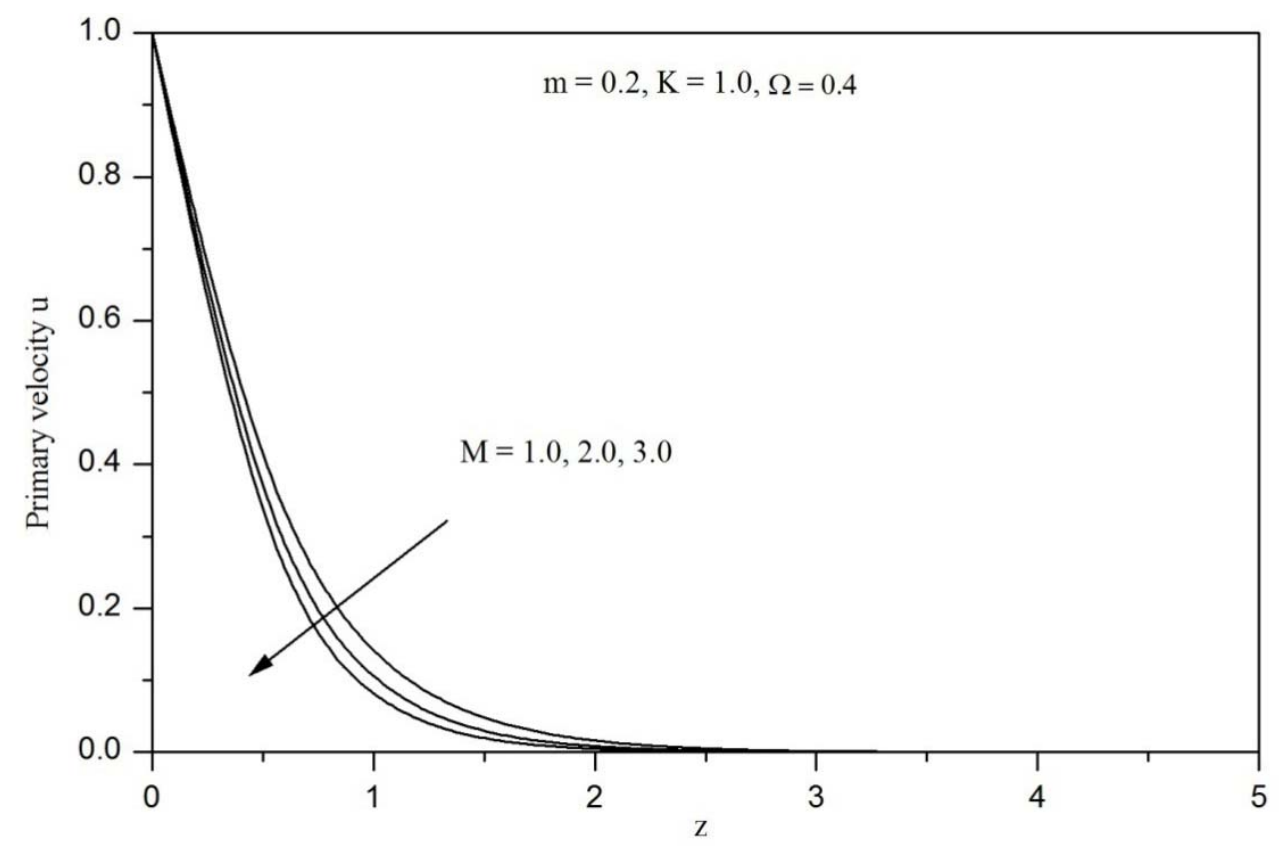

Fig.1. Effect of the magnetic parameter $M$ on the primary velocity. 


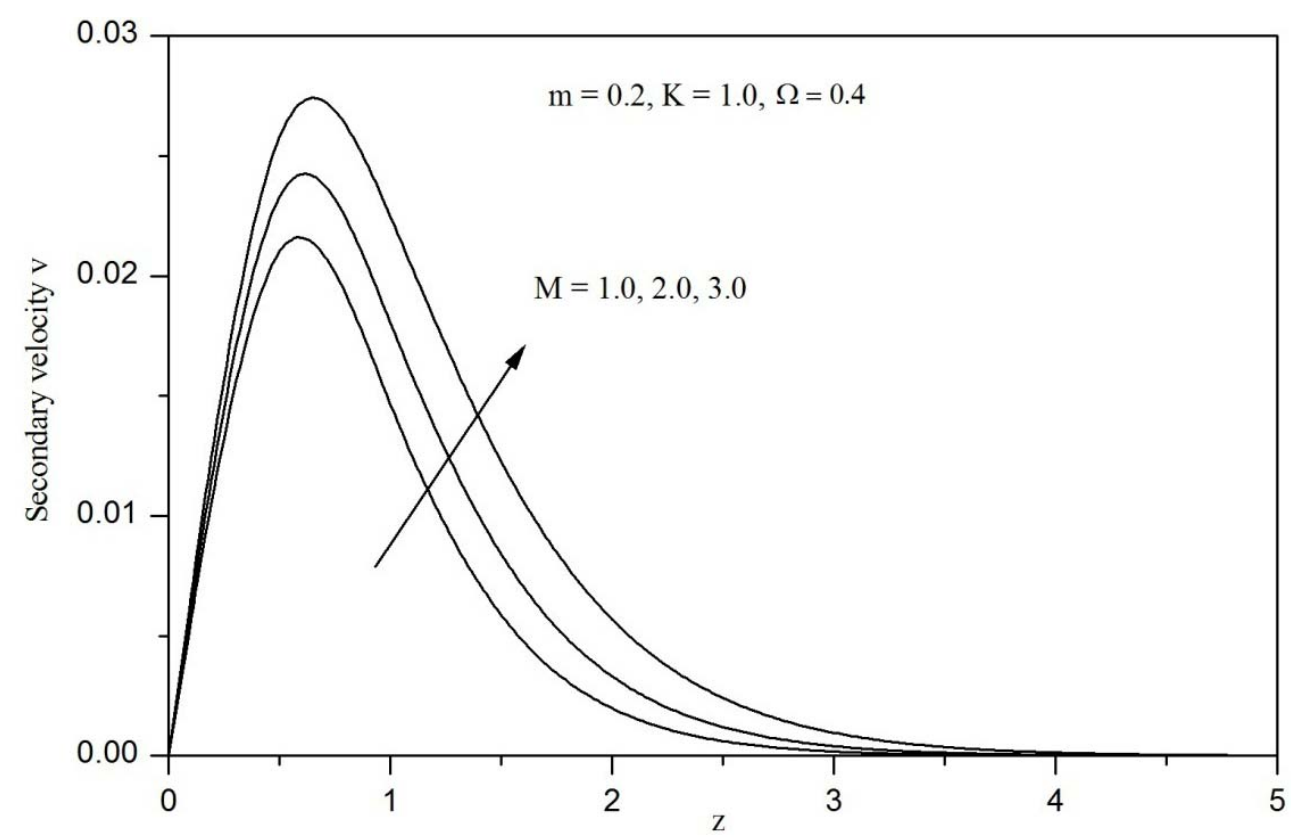

Fig.2. Effect of the magnetic parameter $M$ on the secondary velocity.

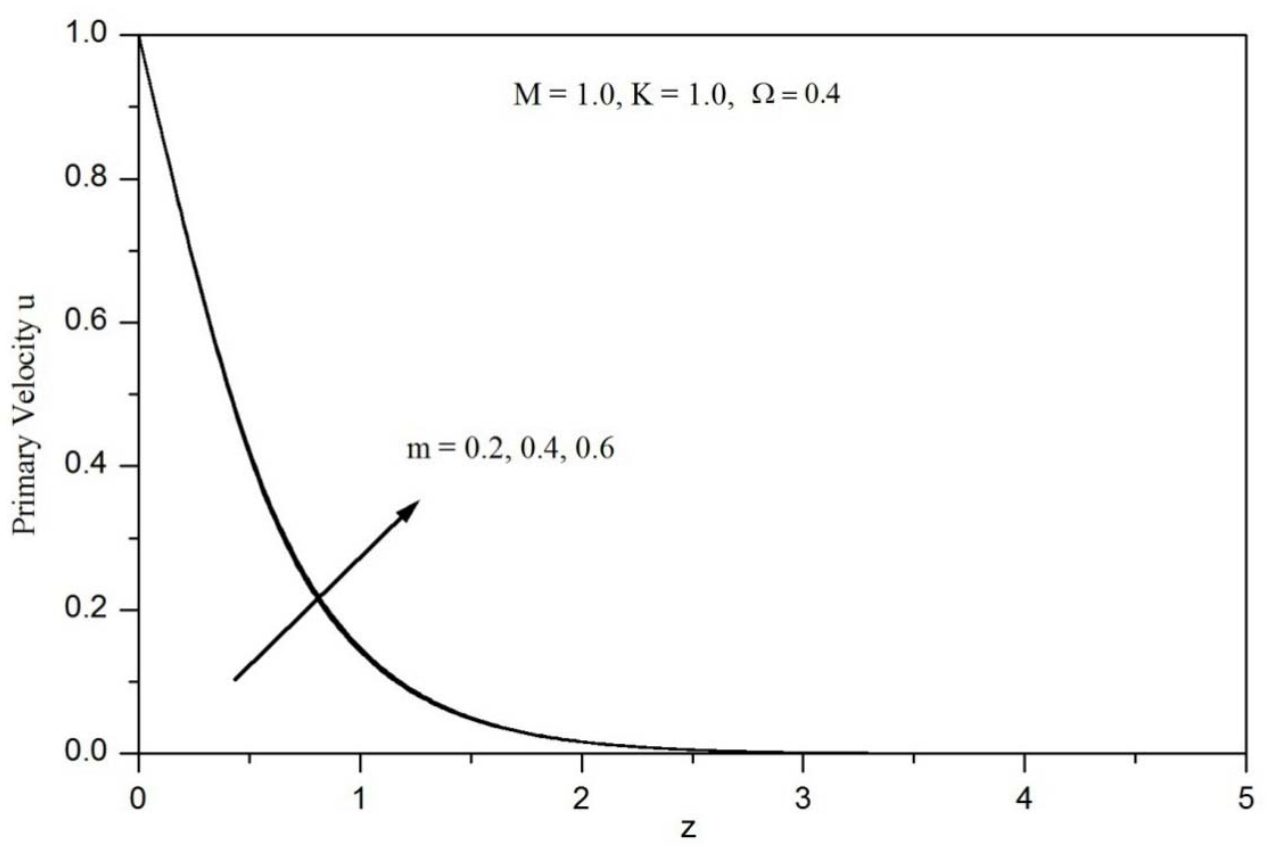

Fig.3. Effect of the Hall parameter $m$ on the primary velocity. 


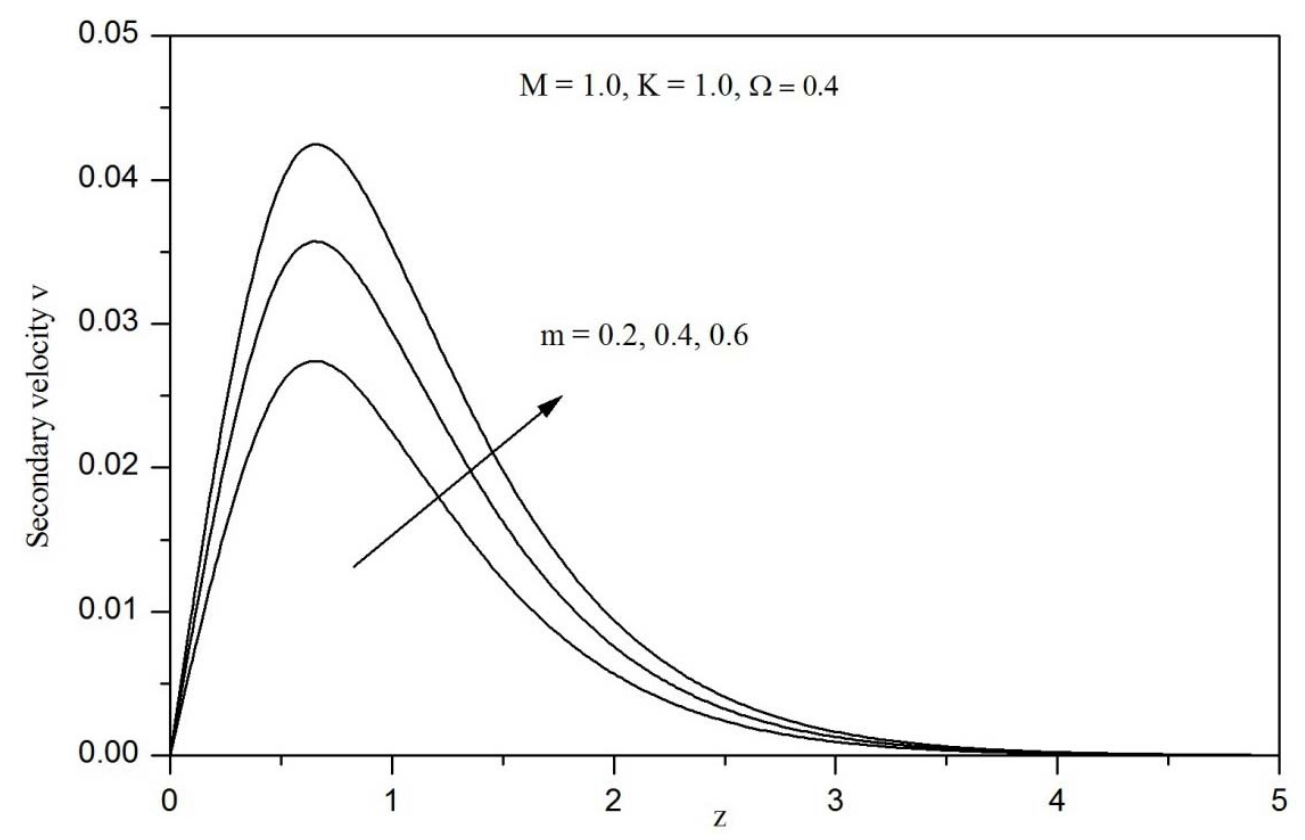

Fig.4. Effect of the Hall parameter $m$ on the secondary velocity.

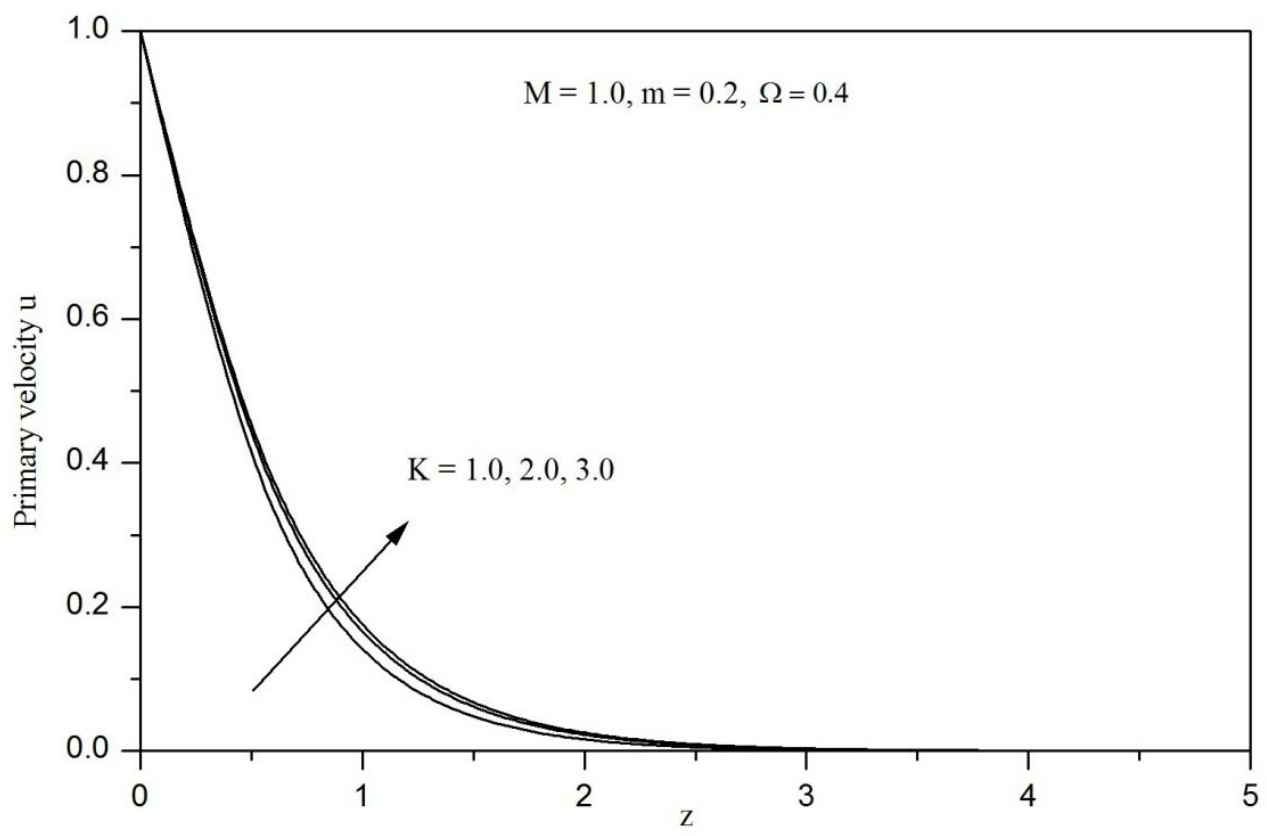

Fig.5. Effect of the porosity parameter $K$ on the primary velocity. 


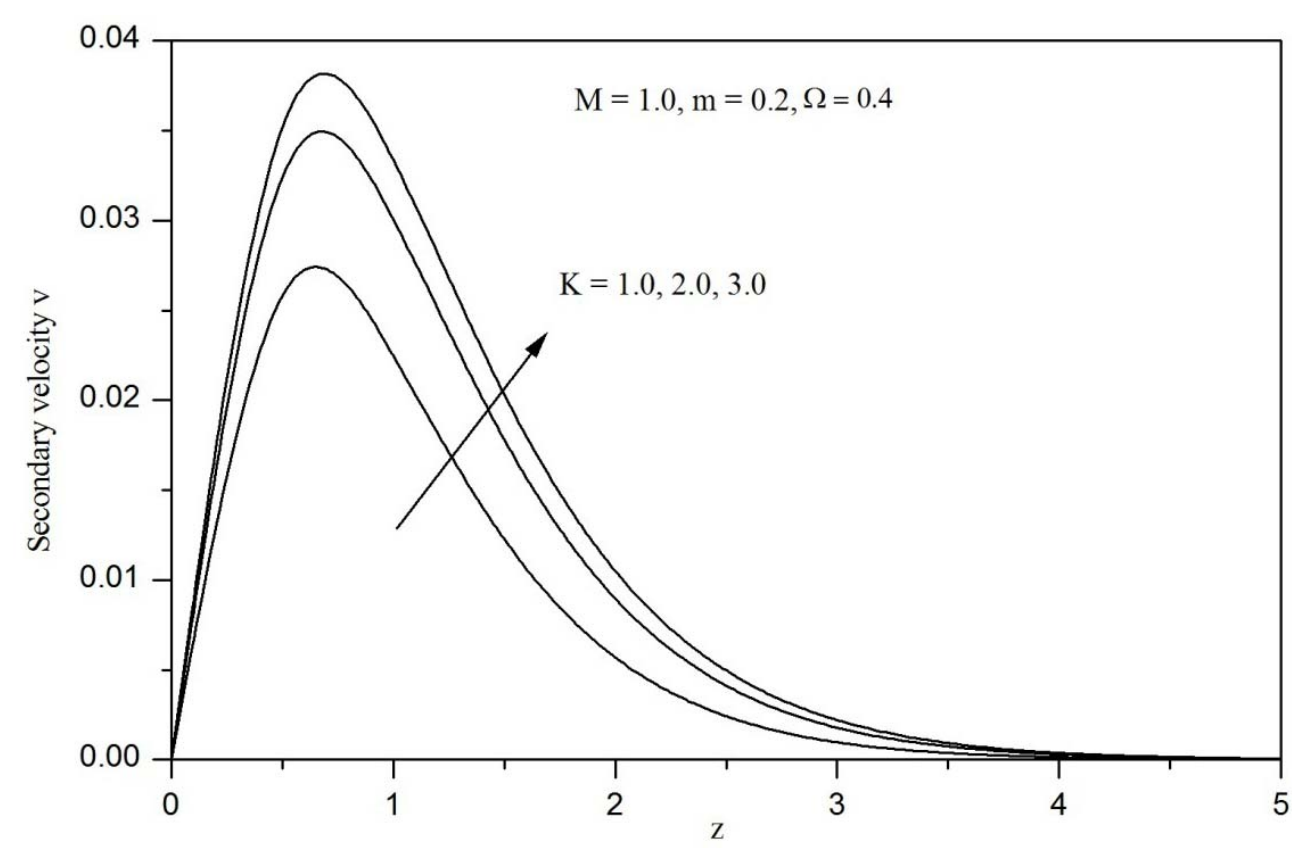

Fig.6. Effect of the porosity parameter $K$ on the secondary velocity.

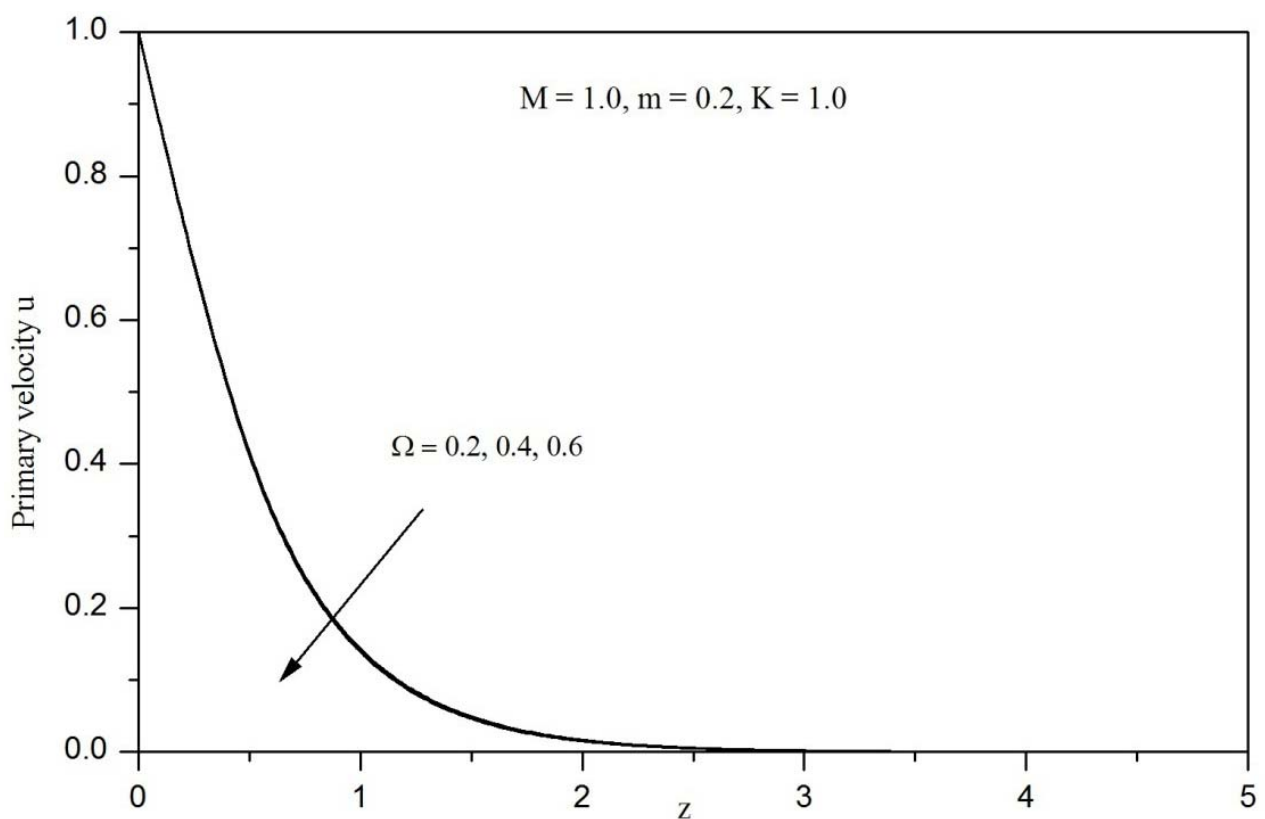

Fig.7. Effect of the rotation parameter $\Omega$ on the primary velocity. 


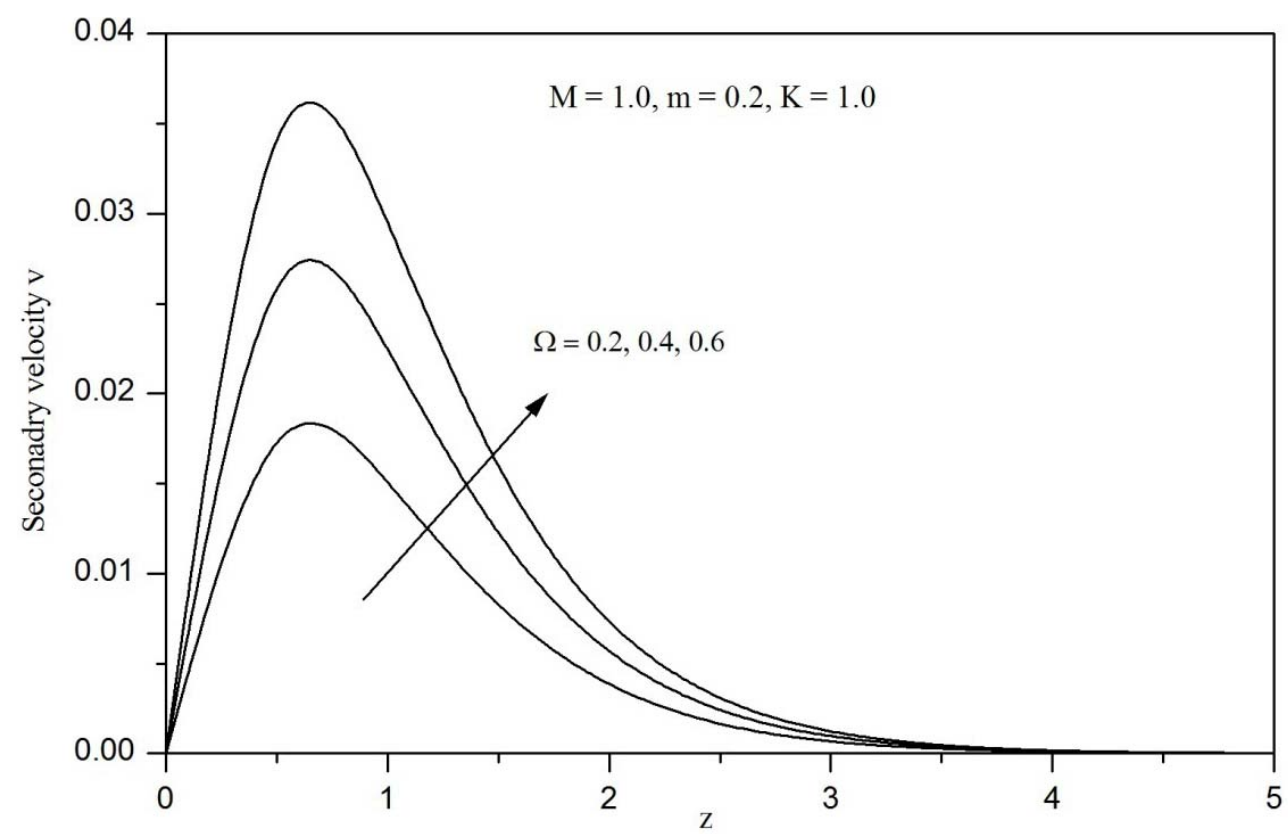

Fig.8. Effect of the rotation parameter $\Omega$ on the secondary velocity.

Table 1. The numerical values of the shearing stresses $\tau_{x}$ and $\tau_{y}$ due to the primary and secondary velocities for different values of $M, m, K$ and $\Omega$.

\begin{tabular}{|l|l|l|l|l|l|}
\hline$M$ & $m$ & $K$ & $\Omega$ & $\tau_{x}$ & $\tau_{y}$ \\
\hline & & & & & \\
1.0 & 0.2 & 1.0 & 0.4 & 1.312586 & 0.066124 \\
2.0 & 0.2 & 1.0 & 0.4 & 1.425732 & 0.067201 \\
1.0 & 0.4 & 1.0 & 0.4 & 1.303914 & 0.085820 \\
1.0 & 0.2 & 2.0 & 0.4 & 1.240932 & 0.081876 \\
1.0 & 0.2 & 1.0 & 0.6 & 1.318460 & 0.087432 \\
\hline
\end{tabular}

\section{Conclusions}

In this paper, an MHD transient three dimensional flow of an electrically conducting viscous incompressible fluid past an impulsively started infinite horizontal porous plate relative to a rotating system has been presented. It is assumed that the entire system rotates with a constant angular velocity about the normal to the plate and a uniform magnetic field is applied along the normal to the plate and directed into the fluid region. The Galerkin FEM has been adopted to solve the non-dimensional governing equations of the flow. The study has shown that the primary velocity is retarded under the effects of the magnetic field and rotation of the fluid, whereas this motion is accelerated under the Hall effect. The secondary velocity is accelerated under the effects of the Hall current and rotation due to the application of the transverse magnetic field. The viscous drag on the plate due to primary motion is reduced under the Hall effect, but this frictional force increases under the effects of the magnetic field and rotation. The shearing stress $\tau_{y}$ due to the secondary velocity rises under the effects of the Hartmann number $M$ and the angular velocity $\Omega$. 


\section{Nomenclature}

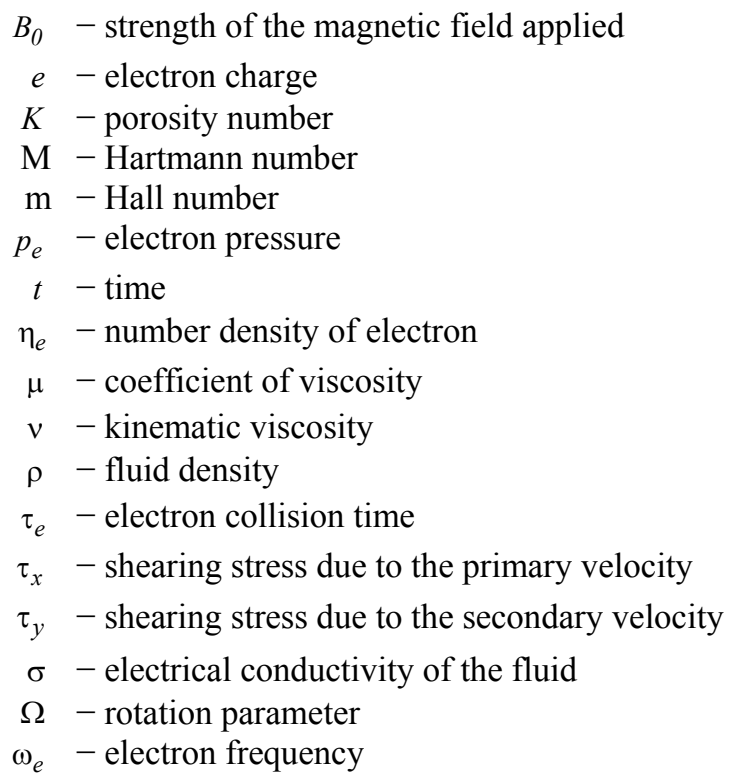

\section{Aknowledgement}

The author wishes to express sincere thanks to the editor and referee for careful reading of the manuscript and valuable suggestions.

\section{References}

[1] Datta N. and Jana R.N. (1976): Oscillatory magneto-hydrodynamic flow past a flat plate with Hall effects. -J. of Physical Society of Japan, vol.40, No.5, pp.1469-1474.

[2] Biswal S. and Sahoo P.K. (1994): Hall effect on oscillatory hydro-magnetic free convective flow of a visco-elastic fluid past an infinite vertical porous flat plate with mass transfer. -Proc. Nat. Acad. Sci, vol.69A, pp.46-52.

[3] Watanabe T. and Pop I. (1995): Hall effects on magneto-hydrodynamic boundary layer flow over a continuous moving flat plate. -Acta Mechanica, vol.108, No.1, pp.35-47.

[4] Aboeldahab E.M. and Elbarbary E.M.E. (2001): Hall current effect on magneto- hydrodynamic free convection flow past a semi-infinite vertical plate with mass transfer. -Int. J. Eng. Science, vol.39, pp.1641-1652.

[5] Acharya M., Dash G.C. and Singh L.P. (2001): Hall effect with simultaneous thermal and mass diffusion on unsteady hydro-magnetic flow near an accelerated vertical plate. - Indian J. of Physics, vol.75B, No.1, pp.168176.

[6] Sharma B.K., Jha A.K and Chaudhary R.C. (2007): Hall effects on MHD mixed convective flow of a viscous incompressible fluid past a vertical porous plate, immersed in a porous medium with heat source/sink. - Rom. Journal Phys, vol.52, No.5-7, pp.487-503.

[7] Prabhakar Reddy B. and Anand Rao J. (2011): Radiation and thermal diffusion effects on an unsteady MHD free convection mass transfer flow past an infinite vertical porous plate with Hall current and heat source. - J. of Eng. Phys. and Thermophysics, vol.84, No.6, pp.1369-1378.

[8] Raju M.C. Varma S.V.K. and Ananad Reddy N. (2011): Hall current effects on unsteady MHD flow between stretching sheet and an oscillating porous upper parallel plate with constant suction. - Thermal Science, vol.15, No.2, pp.45-48. 
[9] Rajput U.S. and Neetu Kanaujia (2016): MHD flow past a vertical plate with variable temperature and mass diffusion in the presence of Hall current. -Int. J. of Applied Sci. and Eng., vol.14, No.2, pp.115-123.

[10] Abdus Sattar M.D. and Abdul Maleque M.D. (2000): Unsteady MHD natural convection flow along an accelerated porous plate with Hall current and mass transfer in a rotating porous medium. - J. Energy, Heat and Mass Transfer, vol.22, pp.67-72.

[11] Takhar H.S., Chamkha A.J. and Nath G. (2002): MHD flow over a moving plate in a rotating fluid with magnetic field, Hall currents and free stream velocity. - Int. J. Eng. Sci, vol.40, No.13, pp.1511-1527.

[12] Mbeledogu I.U. and Ogulu A. (2007): Heat and mass transfer of an unsteady MHD natural convection flow of a rotating fluid past a vertical porous flat plate in the presence of radiative heat transfer. - Int. J. Heat and Mass Transfer, vol.50, pp.1902 -1908.

[13] Singh K.D. and Rakesh Kumar (2009): Combined effects of Hall current and rotation on free convection MHD flow in a porous channel. - Ind. J. of Pure and Appl. Phys., vol.47, pp.617-623.

[14] Ahmed N. and Sarma H.K. (2011): MHD transient flow past an impulsively started infinite horizontal porous plate in a rotating system with Hall current. - Int. J. of Appl. Math. and Mech., vol.7, No.2, pp.1-15.

[15] Satyanarayana P.V., Venkateswarlu B. and Venkataramana S. (2013): Effects of Hall current and radiation absorption on MHD micropolar fluid in a rotating system. -Ain Shams Engineering Journal, vol.4, No.4, pp.843854.

Received: February 23, 2017

Revised: December 18, 2017 